\title{
Autocrine stimulation of VEGFR-2 activates human leukemic cell growth and migration
}

\author{
Sergio Dias, ${ }^{1}$ Koichi Hattori, ${ }^{1,2}$ Zhenping Zhu, ${ }^{3}$ Beate Heissig, ${ }^{2}$ Margaret Choy, ${ }^{1}$ \\ William Lane, ${ }^{1}$ Yan Wu, ${ }^{3}$ Amy Chadburn, ${ }^{4}$ Elizabeth Hyjek, ${ }^{4}$ Muhammad Gill, ${ }^{1}$ \\ Daniel J. Hicklin, ${ }^{3}$ Larry Witte, ${ }^{3}$ M.A.S. Moore, ${ }^{2}$ and Shahin Rafii ${ }^{1}$
}

${ }^{1}$ Weill Medical College of Cornell University, Hematology/Oncology Division, New York, New York, USA

${ }^{2}$ Sloan Kettering Cancer Institute, New York, New York, USA

${ }^{3}$ ImClone Systems Inc., New York, New York, USA

${ }^{4}$ Weill Medical College of Cornell University, Division of Pathology, New York, New York, USA

Address correspondence to: Shahin Rafii, Division of Hematology/Oncology, Room C-606, 1300 York Avenue, New York, New York 10021, USA. Phone: (212) 746-2070; Fax: (212) 746-8866; E-mail: srafii@mail.med.cornell.edu.

Received for publication November 23, 1999, and accepted in revised form July 11, 2000.

\begin{abstract}
Emerging data suggest that VEGF receptors are expressed by endothelial cells as well as hematopoietic stem cells. Therefore, we hypothesized that functional VEGF receptors may also be expressed in malignant counterparts of hematopoietic stem cells such as leukemias. We demonstrate that certain leukemias not only produce VEGF but also express functional VEGFR-2 in vivo and in vitro, resulting in the generation of an autocrine loop that may support leukemic cell survival and proliferation. Approximately $50 \%$ of freshly isolated leukemias expressed mRNA and protein for VEGFR-2. VEGF 165 induced phosphorylation of VEGFR-2 and increased proliferation of leukemic cells, demonstrating these receptors were functional. $\mathrm{VEGF}_{165}$ also induced the expression of MMP9 by leukemic cells and promoted their migration through reconstituted basement membrane. The neutralizing $\mathrm{mAb}$ IMC-1C11, specific to human VEGFR-2, inhibited leukemic cell survival in vitro and blocked VEGF 165 -mediated proliferation of leukemic cells and VEGF-induced leukemic cell migration. Xenotransplantation of primary leukemias and leukemic cell lines into immunocompromised nonobese diabetic mice resulted in significant elevation of human, but not murine, VEGF in plasma and death of inoculated mice within 3 weeks. Injection of IMC-1C11 inhibited proliferation of xenotransplanted human leukemias and significantly increased the survival of inoculated mice. Interruption of signaling by VEGFRs, particularly VEGFR-2, may provide a novel strategy for inhibiting leukemic cell proliferation.
\end{abstract}

J. Clin. Invest. 106:511-521 (2000).

\section{Introduction}

During embryonic development, hematopoietic and early endothelial cells (angioblasts) originate from a common precursor cell known as hemangioblast. Given this common origin, several signaling pathways are shared by both hematopoietic and vascular cells. One such pathway is the VEGFR-2 signaling pathway. VEGFR-2 (KDR, human homologue; Flk-1, murine homologue) binds to several soluble factors including VEGF, which exerts proliferative and migratory effects on endothelium. VEGFR-2 was thought to be exclusively expressed by adult endothelial cells; however, it was only recently shown to be present on a subset of multipotent hematopoietic stem cells (1). Later studies further revealed that certain leukemic cells also expressed VEGFR-2 (2). Considering this new evidence, neoplastic transformation of hematopoietic stem cells into malignant leukemic cells may be associated with recapitulated expression of hemangioblast-associated signaling tyrosine kinases such as VEGFR-2. Because VEGF is also produced by leukemic cells (2-4), coinciding expression of VEGF receptors may result in the generation of an autocrine loop that supports the proliferation and survival of leukemic cells.

The two primary signaling tyrosine kinase receptors that mediate the various biologic effects of VEGF are VEGFR-2 and VEGFR-1 (Flt-1). Although the binding affinity of VEGFR-1 to VEGFR is very high, with IC $_{50}$ values of 10-70 pM (5), most studies have shown that VEGFR-2 is the critical receptor for transmitting cellular signals for the proliferation and differentiation of endothelial cells (6), whereas VEGFR-1 may be more important for vascular remodeling. The relative significance of VEGF receptors in the regulation of vasculogenesis and angiogenesis has been established in studies in which the VEGFR-2 and VEGFR-1 genes were disrupted in murine embryonic stem cells by homologous recombination. Mice deficient in VEGFR-2 had drastic defects in vasculogenesis, angiogenesis, and hematopoiesis (7). In contrast, VEGFR-1 knockout mice developed abnormal vascular channels, suggesting a role for this receptor in the regulation of endothelial cell-cell or cell-matrix interactions (8). Disruption 
of VEGFR-2 signaling resulted in inhibition of tumor growth and tumor metastasis. In fact, neutralizing $\mathrm{mAb}$ to murine VEGFR-2 inhibited tumor growth and metastasis in murine models $(9,10)$. Furthermore, glioblastoma growth was inhibited in mice dominantnegative for VEGFR-2 (11).

Leukemias originate from hematopoietic stem cells at different stages of their maturation and differentiation. It is now well established that acute leukemias originate from immature hematopoietic stem cells that have the capacity to undergo self-renewal, whereas less aggressive leukemias such as chronic leukemias seem to originate from the more mature committed hematopoietic progenitor cells. Several studies have shown that VEGF is almost invariably expressed by all established leukemic cell lines, including the well-studied HL-60 leukemic cell line $(2,3)$, as well as freshly isolated human leukemias. Using RT-PCR, several studies have also shown that VEGFR-2 and VEGFR-1 are expressed by certain human leukemias $(2,3)$. However, none of these studies have shown whether expression of these receptors is associated with any functional response.

In this report, we demonstrate that VEGF receptors expressed on leukemic cells are functional and convey signals similar to those on endothelial cells such as increasing proliferation, MMP activation, and transbasement membrane migration. Moreover, using a neutralizing mAb specific for human VEGFR-2, we demonstrate that inhibition of VEGFR-2 either in vitro or in vivo inhibits proliferation of VEGFR-2+ human leukemias. Therefore, blocking VEGFR-2 signaling may represent a novel therapeutic approach for the treatment of a subset of acute leukemias.

\section{Methods}

All chemicals and reagents were obtained from Sigma Chemical Co. (St. Louis, Missouri, USA) unless stated otherwise.

Monoclonal neutralizing antibodies to VEGF receptors. We used an immunoneutralizing $\mathrm{mAb}$ to KDR/VEGFR2, IMC-1C11 (ImClone Systems Inc.), to block VEGF/VEGFR-2 interactions in vitro and in vivo. A neutralizing $\mathrm{mAb}$ to VEGFR-1/Flt-1 was also used in vitro (clone 6.12; ImClone Systems Inc.). These antibodies have been previously shown to block VEGFinduced endothelial cell proliferation and receptor phosphorylation $(12,13)$. Importantly, IMC-1C11 has been shown not to cross react with the murine homologue of KDR, flk-1 (14).

Collection and culture of primary lenkemia samples. Peripheral blood samples from leukemic patients (diagnosed with acute leukemia) were collected by phlebotomy, diluted 1/2 in Hanks' buffered saline (Life Technologies Inc., Grand Island, New York, USA), and overlaid on $5 \mathrm{~mL}$ of Lymphoprep (Ficoll; Accurate Chemical and Scientific Corp., Westbury, New York, USA). Each sample was spun at 2,000 $\mathrm{g}$ for 30 minutes, and the mononuclear cell interphase was collected into a fresh tube and washed twice with Hanks' for 5 minutes at $780 \mathrm{~g}$. The resulting cell pel- let was finally resuspended in RPMI/10\% FCS and cultured overnight in RPMI/10\% FCS, to eliminate possible contamination with monocytes/macrophages. These will adhere readily to the tissue culture flasks, and the cells remaining in suspension consist mainly of leukemic blasts. These leukemic cells were subsequently transferred to another flask and, before the addition of VEGF and/or VEGFR antibodies, were serum starved for 16 hours overnight, in serum free RPMI, as already described here.

Culture of lenkemic cell lines. The three leukemic cell lines used in this study, HL-60 (pro-myelomonocytic), HEL (megakaryocytic), and K562 (CML/erythroid) were cultured in RPMI with 10\% FCS, penicillin (100 $\mathrm{U} / \mathrm{mL})$, streptomycin $(100 \mu \mathrm{g} / \mathrm{mL})$, and fungizone $(0.25 \mu \mathrm{g} / \mathrm{mL})$. Before incubation with VEGF and/or antibodies, cells were serum starved for 16 hours in RPMI alone. For proliferation experiments, cells were cultured in six-well plates (Corning-Costar Corp., Cambridge, Massachusetts, USA), at a cell density of $1 \times 10^{5}$ cells per well, in serum-free RPMI. Cells were treated (10-50 ng/mL VEGF) or untreated (RPMI alone) and were cultured in the presence or absence of $500 \mathrm{ng}$ to 1 $\mu \mathrm{g} / \mathrm{mL}$ of IMC-1C11, or an mAb to FLT-1/VEGFR-1, clone 6.12. After $24,48,72$, and 96 hours, viable cells (as determined by trypan blue exclusion) were counted in triplicate using a hemocytometer. Each experimental condition was done in triplicate, and experiments with the leukemic cell lines were repeated three times.

$R N A$ extraction, cDNA synthesis, and RT-PCR. Total RNA was extracted using TRI-reagent, following the manufacturer's instructions. cDNA was subsequently synthesized from total RNA using the Ready-to-Go Kit (Amersham Pharmacia Biotech, Piscataway, New Jersey, USA), and PCR was performed using a PCR thermal cycler (MWG Biotech, High Point, North Carolina, USA). The PCR program used to amplify VEGFR-2, VEGFR-1, and $\beta$-actin consisted of a precycle of 5 minutes at $94^{\circ} \mathrm{C}, 45$ seconds at $60^{\circ} \mathrm{C}$, and 45 seconds at $72^{\circ} \mathrm{C}$. After this initial cycle, the reaction was continued for 35 cycles of 1 minute at $94^{\circ} \mathrm{C}, 45$ seconds at $65^{\circ} \mathrm{C}$, and 2 minutes at $72^{\circ} \mathrm{C}$ and concluded with 7 minutes at $72^{\circ} \mathrm{C}$. The primer sequences were as follows: $\beta$-actin forward primer: TCATGTTTGAGACCTTCAA; $\beta$ actin reverse primer: GTCTTTGCGGATGTCCACG ( $\beta$ actin PCR product: $513 \mathrm{bp}$ ); VEGFR-2 forward primer: GTGACCAACATGGAGTCGTG; VEGFR-2 reverse primer: CCAGAGATTCCATGCCACTT (VEGFR-2 PCR product: $660 \mathrm{bp}$ ); VEGFR-1 forward primer: ATTTGTGATTTTGGCCTTGC; VEGFR-1 reverse primer: CAGGCTCATGAACTTGAAAGC (VEGFR-1 PCR product: $550 \mathrm{bp}$ ). Endothelial cell cDNA was used as positive control for all three sets of primers.

Protein extraction and Western blotting. Phosphorylated VEGF receptors (VEGFR-1 and VEGFR-2) were detected by Western blotting, after cell incubation with 20 $\mathrm{ng} / \mathrm{mL} \mathrm{VEGF}_{165}$ (R\&D Systems Inc., Minneapolis, Minnesota, USA) for 10 minutes at $37^{\circ} \mathrm{C}$. After this brief stimulation, total protein extracts from primary leukemic cells and cell lines were obtained by lysing the 
cells in cold RIPA buffer (50 mM Tris, 5 mM EDTA, 1\% Triton X-114, 0.4\% sodium cacodylate, and $150 \mathrm{mM}$ $\mathrm{NaCl})$, in the presence of protease inhibitors $(1 \mathrm{mg} / \mathrm{mL}$ aprotinin, $10 \mathrm{mg} / \mathrm{mL}$ leupeptin, $1 \mathrm{mM} \beta$-glycerophosphate, $1 \mathrm{mM}$ sodium orthovanadate, and $1 \mathrm{mM}$ PMSF). Embryonic fibroblasts (the MRC5 cell line) were used as negative control. After centrifugation to remove cell debris, supernatants (a total protein minimum of 500 ng) were immunoprecipitated overnight at $4^{\circ} \mathrm{C}$ with protein-G agarose beads and an anti-phosphotyrosine antibody (Santa Cruz Biotechnology Inc., Santa Cruz, California, USA) to precipitate phosphorylated proteins. These were resuspended in loading buffer and subjected to SDS-PAGE-acrylamide gel electrophoresis (7.5\% gels) under reducing conditions (in the presence of $\beta$-mercaptoethanol). Proteins were subsequently blotted onto a nitrocellulose membrane following conventional protocols. Finally, blots were blocked in $1 \%$ BSA/PBS-1\% Tween-20 for 1 hour at room temperature followed by incubation with primary and secondary antibodies. Rabbit polyclonal anti-VEGFR-2 (Santa Cruz Biotechnology Inc.) and goat monoclonal antiVEGFR-1 (R\&D Systems Inc.) antibodies were used at a concentration of $1 \mu \mathrm{g} / \mathrm{mL}$, and secondary anti-rabbit IgG-HRP (for VEGFR-2) or anti-goat IgG-HRP (for VEGFR-1) were used at 1:6,000. The ECL chemiluminescence detection system and ECL film (Amersham Pharmacia Biotech) were used to visualize the presence of proteins on the nitrocellulose blots.

Gelatinolytic zymography. Supernatants from leukemic cell lines and primary leukemia cultures were collected after overnight incubation in serum-free medium, with or without $\mathrm{VEGF}_{165}$, and their metalloproteinase activity was measured by gelatinolytic zymography, as described previously (15). Briefly, cell culture supernatants were treated with gelatin-agarose beads, to concentrate the gelatinases, and processed through SDSPAGE-acrylamide gels containing $1 \%$ gelatin. The gels were subsequently incubated in $2.5 \%$ Triton X-100 for 1 hour at room temperature, rinsed in distilled water, and placed in low-salt collagenase buffer $(50 \mathrm{mM}$ Tris $[\mathrm{pH}$ 7.6], 0.2 $\mathrm{M} \mathrm{NaCl}, 5 \mathrm{mM} \mathrm{CaCl}_{2}$, and $0.2 \% \mathrm{vol} / \mathrm{vol}$ Brij-35) at $37^{\circ} \mathrm{C}$ for 18 hours. Bands of gelatinolytic activity were visualized after staining the gels with $10 \mathrm{~mL}$ of a $0.2 \%$ Coomassie blue solution and $190 \mathrm{~mL}$ destain (distilled water, methanol, and glacial acetic acid; 6:3:1) for 30 minutes to 1 hour at room temperature. For each experiment, supernatants from $1 \times 10^{6}$ cells were collected, and each experiment was done in triplicate. The Adobe Photoshop 4.0 software application (Adobe Systems, Mountain View, California, USA) and a Umax Astra scanner (Umax, Fremont, California, USA) were used to scan the gels and the intensity of the gelatinolytic bands was assessed using NIH Image (version 1.58).

Migration experiments. Freshly isolated leukemic cells and cell lines were resuspended in serum-free RPMI, and a stock of $10^{6}$ cells $/ \mathrm{mL}$ was prepared. A modified version of a transwell migration technique described previously (16) was used. Briefly, leukemic cell aliquots
$(100 \mu \mathrm{L})$ were added to $8-\mu \mathrm{m}$-pore transwell inserts, coated with $25 \mu \mathrm{g}$ of growth factor-depleted Matrigel (Becton Dickinson Immunocytometry Systems, San Jose, California, USA), and placed into the wells of a 24well plate. The lower compartment contained serumfree RPMI with or without $200 \mathrm{ng} / \mathrm{mLVEGF}_{165}$. For the purpose of blocking migration, each condition was prepared in a separate aliquot and incubated with IMC$1 \mathrm{C} 11$ ( $1 \mu \mathrm{g} /$ condition), anti-VEGFR-1 (clone 6.12; 1 $\mu \mathrm{g} /$ condition), or rhTIMP-1 (Oncogene Research Products, Cambridge, Massachusetts, USA). The migration was carried out at $37^{\circ} \mathrm{C}$ and $5 \% \mathrm{CO}_{2}$ for $14-18$ hours. Migrated cells were collected from the lower compartment, spun down at $4650 \mathrm{~g}$ and counted using a hemocytometer. Only live cells, as determined by trypan blue exclusion, were considered in the quantification. Experiments were done in triplicate, and results are shown as the number of cells migrated in response to VEGF.

Detection of KDR/VEGFR-2, FLT-1/VEGFR-1, and VEGF on ectopically implanted leukemias (chloromas) by immunobistochemistry. Paraffin-embedded chloroma sections were immunohistochemically stained for VEGFR-1 and VEGFR-2, following conventional protocols. The antibodies used were mouse mAb to VEGFR-2/Flk-1 (Santa Cruz Biotechnology Inc.) used at $300 \mathrm{ng} / \mathrm{mL}$; rabbit polyclonal antibody to VEGFR-1 (R\&D Systems Inc.), used at $200 \mathrm{ng} / \mathrm{mL}$; vWF polyclonal antibody, used at $200 \mathrm{ng} / \mathrm{mL}$; and VEGF polyclonal antibody (Zymed Laboratories Inc., South San Francisco, California, USA), used at $200 \mathrm{ng} / \mathrm{mL}$. For single immunohistochemical staining, peroxidase-labeled secondary antibodies (against mouse and rabbit immunoglobulins) were used at a $1 / 6,000$ dilution. For VEGF/VEGFR-2 double immunostaining, two detection systems were used. Incubation with anti-VEGFR2/Flk-1 was followed by goat anti-mouse IgG (1:200) and PAP complexes. The peroxidase reaction was devel-

\section{Table 1}

VEGF production and VEGF receptor expression of three leukemic cell lines (HL-60, HEL, and K562) and ten primary leukemia samples (samples 1-10)

\begin{tabular}{lrcc} 
Leukemic cells & $\begin{array}{r}\text { VEGF } \\
(\mathrm{pg} / \mathrm{mL})\end{array}$ & $\begin{array}{c}\text { VEGFR-1 } \\
(\mathrm{mRNA})\end{array}$ & $\begin{array}{c}\text { VEGFR-2 } \\
(\mathrm{mRNA})\end{array}$ \\
HL-60 & $\begin{array}{r}144 \pm 35 \\
94 \pm 15\end{array}$ & + & + \\
HEL & $120 \pm 16$ & + & + \\
Sample 1 & $196 \pm 20$ & + & + \\
Sample 2 & $185 \pm 16$ & + & + \\
Sample 3 & $192 \pm 25$ & + & + \\
Sample 4 & $100 \pm 20$ & + & + \\
Sample 5 & $85 \pm 28$ & - & - \\
K562 & $88 \pm 18$ & + \\
Sample 6 & $90 \pm 12$ & + & - \\
Sample 7 & $93 \pm 21$ & + & - \\
Sample 8 & $77 \pm 14$ & + & - \\
Sample 9 & $78 \pm 22$ & + & - \\
Sample 10 & & + & - \\
\hline
\end{tabular}

VEGF production was determined by ELISA. VEGF receptor expression was determined by RT-PCR. See Methods. 

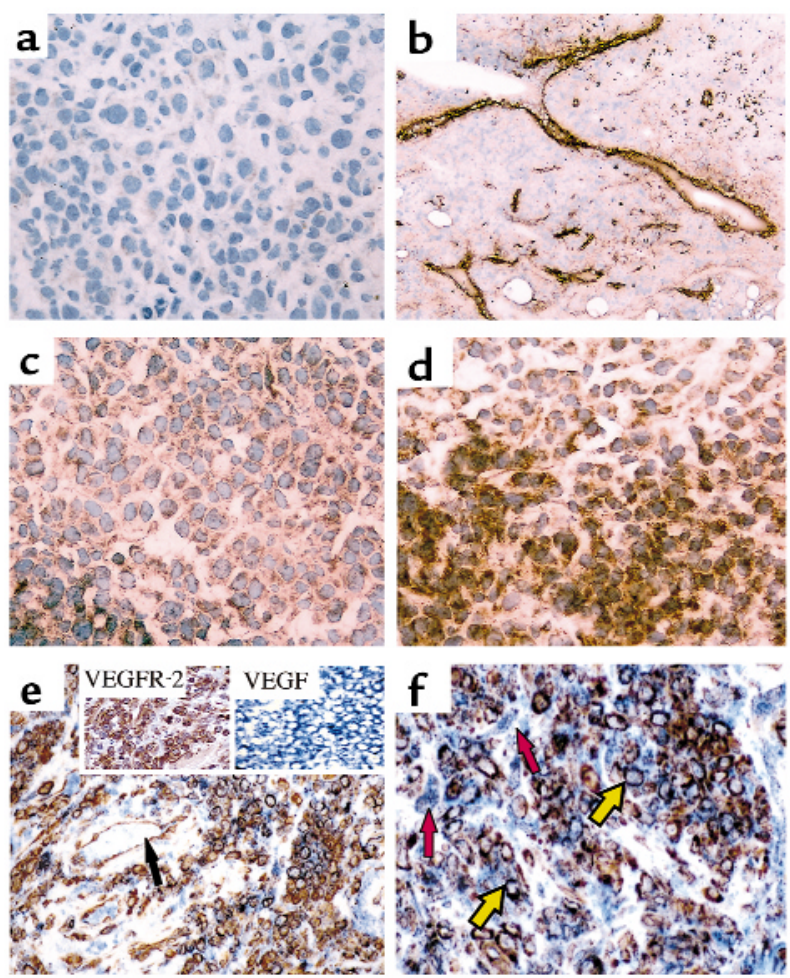

Figure 1

Human chloromas express FLT-1/VEGFR-1 and KDR/VEGFR-2. Sections from human chloromas were stained by immunohistochemistry, as described in Methods. (a) Control IgG, showing little nonspecific staining $(\times 400)$; (b) factor VIII staining, showing specific blood vessel staining $(\times 100)$; (c) KDR/VEGFR-2 staining, on leukemic cell areas $(\times 400)$; (d) FLT-1/VEGFR-1 expression, also detected on a subset of leukemic cells $(\times 400)$. (e and $\mathbf{f})$ Double immunohistochemical staining, showing leukemic cells stain for VEGFR-2 and VEGF (red arrows). Stromal cells stain for VEGF (yellow arrows), whereas endothelial cells stain solely for VEGFR-2 (black arrows). $\times 400$. Insets show stain for VEGFR- 2 alone or VEGF alone. These results are representative of four different samples.

oped with a diaminobenzidine substrate. Immunodetection of VEGF was demonstrated using the Vector blue alkaline phosphatase substrate (Vector Laboratories Inc., Burlingame, California, USA). Sections with single immunohistochemical staining were counterstained with hematoxylin and eosin. All sections were observed under a light microscope.

In vivo experiments with the HL-60 and HEL cell lines and a primary lenkemia. Nonobese diabetic immunocompromised (NOD-SCID) mice were used in all experiments. These were age and sex matched. In the beginning of each experiment, HL-60 $\left(3 \times 10^{6} /\right.$ mouse $)$, HEL $\left(5 \times 10^{6} /\right.$ mouse $)$, or primary leukemia $\left(5 \times 10^{6} /\right.$ mouse $)$ cells were injected (intravenously) into 24 NOD-SCID mice, and 4 days after injection the mice were divided into three groups of eight mice. One group was treated intraperitoneally with IMC-1C11 (400 $\mu \mathrm{g}$ three times a week), whereas the control group was injected with $\mathrm{PBS} / 1 \% \mathrm{BSA}$ (diluent control for the antibody), and a third group was treated intraperitoneally with $400 \mu \mathrm{g}$ of unrelated human IgG (Bayer Corp., Elkhart, Indiana, USA) for the duration of the experiment.

At days 14-15 after the start of the experiment, three mice from each group were sacrificed, their plasma collected into capillary tubes, and their organs collected and processed for histology, to determine the existence of metastasis.

Quantification of VEGF levels in cell culture supernatants and in mouse plasma. An ELISA kit (R\&D Systems Inc.) specific for human VEGF was used, to quantify the levels of VEGF produced by leukemic cells in vitro. Cells were cultured in serum-free conditions, and the culture supernatants collected after 24 hours These were used without further dilution. Each sample was measured in triplicate.

Two ELISA kits (both from R\&D Systems Inc.), specific for human and murine VEGF, were used to determine VEGF concentrations in the plasma of leukemiainoculated mice. Plasma samples were collected at different time points after cell injection and used without any further dilution. Each sample was assayed in triplicate, and the measurements were done in two separate experiments. Both assays have a sensitivity limit of $7.5 \mathrm{pg} / \mathrm{mL}$ and were developed according to the manufacturer's instructions.

Quantification of lenkemic cell engraftment in peripheral blood and bone marrow of NOD-SCID mice. On HL-60and HEL-injected mice, the percentage of human leukemic cells in the bone marrow was quantified by staining for human specific antigens and analyzed by flow cytometry. Briefly, tibias were surgically removed from mice on day 15 after the start of the experiment, and their cellular content was flushed using insulin syringes with 27-gauge needles (Becton Dickinson Immunocytometry Systems) into serumfree medium (RPMI). Bone marrow cells were stained following conventional protocols. In the case of the primary leukemia sample, PBMCs from inoculated mice were analyzed for the presence of human specific markers, following conventional staining protocols. The following antibodies were used: $\mathrm{mAB}$ against human CD15 (PE-labeled, Clone DU-HL603; Sigma Chemical Co.); mAb against human CD45 (PE-labeled, Clone KC56; Coulter Corp., Miami, Florida, USA); mAb against human VEGFR-2 (FITClabeled, clone 6.64; ImClone Systems Inc.); and an isotypic control, MSIgG1 (FITC/PE-labeled, clone 2T8-2F5; Coulter Corp.). The percentage of human leukemic cells in mouse bone marrow and peripher-
Figure 2
Representative RT-PCR analysis for VEGFR-2 and VEGFR-1, on leukemic cell lines. The cell lines HL-60 and HEL express VEGFR-1 and VEGFR-2, as detected by RT- PCR. K562 cells were VEGFR-2 negative. $\beta$-actin was used as an internal control. 


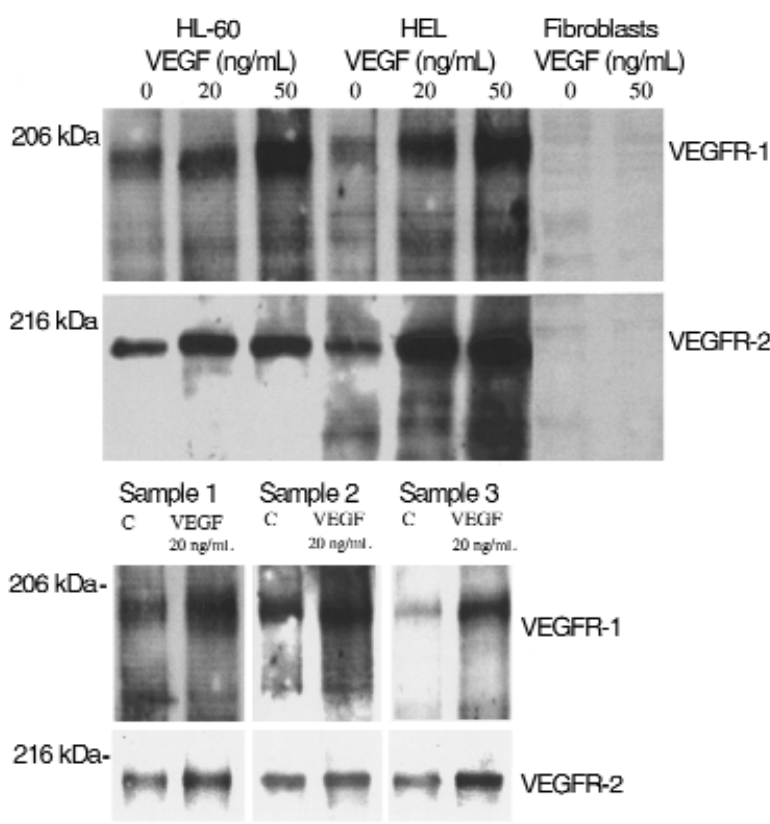

Figure 3

VEGF $_{165}$ induced dose-dependent VEGFR-2 phosphorylation on HL-60 and HEL cells and primary leukemias 1, 2, and 3. Receptor tyrosine phosphorylation was done as described in Methods. Fibroblasts were used as negative controls and showed no sign of VEGFR-2 phosphorylation. These results are representative of three separate experiments. C, control.

al blood samples was determined using a Coulter Elite flow cytometer (Coulter Corp.).

\section{Results}

Human chloromas express VEGF and VEGF receptors. Human leukemias are not only localized to the bone marrow and peripheral circulation but may also infiltrate tissues and form solid masses referred to as chloromas.

Immunohistochemical staining of human chloromas with antibodies specific for VEGFR-1 or VEGFR-2 revealed that, besides staining the endothelial lining of blood vessels, these receptors were also expressed by a subset of the leukemic cells (Figure 1, a and b). The staining localized to the cell membrane, and positive cells appeared scattered throughout the sections (Figure 1, c and d). Overall, there were more VEGFR-2-positive than VEGFR-1-positive areas in the sections analyzed. These receptors were primarily detected in different areas of the tumor, suggesting that the staining pattern of VEGFR1 and VEGFR-2 may identify different cell populations. Furthermore, using a double immunostaining technique, it was shown that, on the sections analyzed, VEGFR-2-positive leukemic cells also stained for VEGF (Figure 1, e and f). Importantly, stromal cells did not stain for VEGFR-2 but expressed VEGF (Figure 1e), whereas endothelium shows a reverse staining pattern, staining for VEGFR-2 but not for VEGF (Figure 1f).

Primary leukemias and lenkemic cell lines produce VEGF and express VEGF receptors. Three leukemic cell lines and ten primary leukemias were analyzed for the produc- tion of VEGF and for the expression of VEGFR-2 by ELISA and RT-PCR, respectively.

All leukemic cells described in this study produced variable amounts of VEGF in vitro, as determined by ELISA (Table 1). However, only $50 \%$ of primary leukemias and two of three cell lines expressed VEGFR-2 at the mRNA level (Figure 2 and Table 1). All VEGFR-2-positive leukemias were also positive for VEGFR-1 (Table 1).

Leukemic cells express functional VEGF receptors. VEGF induced receptor phosphorylation on leukemic cells, as demonstrated by immunoprecipitation and Western blotting, confirming that these are functional, signaling receptors. VEGF 165 induced, in a dose-dependent manner $(20-50 \mathrm{ng} / \mathrm{mL})$, an increase in VEGFR-1 and VEGFR-2 phosphorylation on VEGF receptor-positive leukemic cell lines and primary leukemias, but not on fibroblasts or VEGFR-negative leukemic cells (Figure $3)$. In the absence of exogenous VEGF, leukemic cells had baseline VEGFR-1 and VEGFR-2 phosphorylation (Figure 3), which may be due to the production of VEGF and expression of its receptors by the same cells.

VEGF mediates lenkemic cell proliferation in vitro in an autocrine manner. In the absence of exogenous growth factors (serum-free conditions), leukemic cells show a modest increase in proliferation over a period of 2-3 days (Figure 4a). IMC-1C11 $(1 \mu \mathrm{g} / \mathrm{mL})$ blocked proliferation of VEGFR-2-positive leukemias, such as HL-60, HEL, and primary samples 1 and 2, by $40-60 \%$ after 48-72 hours, but had no effect on VEGFR-2-negative cells such as K562 and primary samples 6 and $7(P<$ 0.01; Figure 4a). mAb to VEGFR-1 (clone 6.12), used at the same concentration, had no effect on leukemic cell proliferation (data not shown). These data show that under serum-free conditions, on VEGF-producing, VEGFR-2-positive leukemias, blockade of VEGFR-2 signaling decreases leukemic cell proliferation.

VEGF induces leukemic cell proliferation, an effect mediated through VEGFR-2. Addition of exogenous VEGF ${ }_{165}$ to leukemic cell cultures increased the proliferation of VEGFR-2-positive leukemias and leukemic cell lines (Figure $4 \mathrm{~b}$ ). IMC-1C11, used at $1 \mu \mathrm{g} / \mathrm{mL}$, blocked this increase in proliferation (Figure $4 \mathrm{~b} ; P<0.05$ ). $\mathrm{mAb}$ to VEGFR-1 (used at the same concentrations) had no effect on $\mathrm{VEGF}_{165}$-induced leukemic cell proliferation (data not shown), suggesting that on these cells the mitogenic effects of VEGF 165 are mediated mainly through VEGFR-2. Addition of exogenous VEGF to VEGFR-2-negative cells, such as the K562 cell line, had no effect on cell proliferation (Figure 4b). Similar results were seen with the VEGFR-2-negative primary leukemic samples 6-10 (data not shown).

VEGF induces MMP secretion/production by lenkemic cells. Without stimulation, in serum-free conditions, leukemic cells release variable amounts of MMPs into the cell culture supernatant (Figure 5). This suggests that MMP production by leukemic cells may identify a leukemic subtype or subpopulation with a more invasive phenotype. On primary leukemias and/or cell lines investigat- 

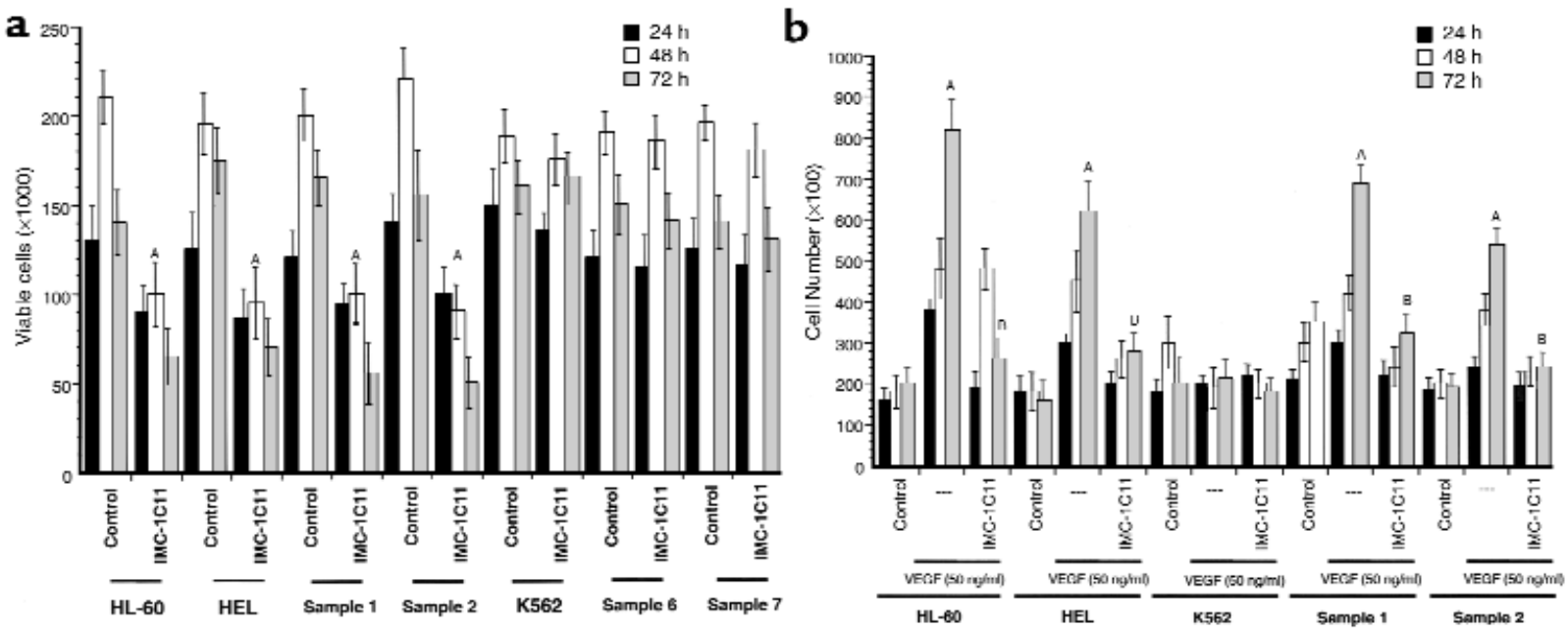

Figure 4

(a) Leukemic cell growth in serum-free media. Treatment of VEGFR-2-positive cells (HL-60, HEL, and primary samples 1 and 2), but not of VEGFR-2-negative cells (K562 and samples 6 and 7), with IMC-1C11 decreases cell growth (AP $<0.005$, at 48 and 72 hours). The results shown are representative of three separate experiments. (b) VEGF 165 induced proliferation of a subset of leukemias, an effect mediated through KDR/VEGFR-2. VEGF 165 induced a significant $\left({ }^{A} P<0.003\right)$ increase in proliferation of all VEGFR-2-positive leukemias (two cell lines and two primary leukemias are shown). This effect was blocked by IMC-1C11 ( $P<<0.04)$, as described in Methods. The results shown are representative of three separate experiments.

ed, the myelo-monocytic subtypes (shown in Figure 5: HL-60 cells, samples 2 and 3) have consistently shown a higher level of basal MMP production and release. Incubation with $V \mathrm{EGF}_{165}$ increased MMP-9 release by leukemic cells (Figure 5a) over an 18-hour period. Quantification of $\mathrm{VEGF}_{165}$-induced increases in secreted MMP-9, by densitometry, indicated that this effect was significant (Figure 5b). On primary leukemias, MMP-9 was the main MMP released into the culture supernatants (Figure 5). The levels of TIMP-1 produced by leukemic cells were also investigated, by Western blotting, but showed only a minor variation after stimulation with $\mathrm{VEGF}_{165}$ (data not shown).

VEGF induces lenkemic cell migration through Matrigelcoated transwells, an effect mediated through VEGFR-1 and $V E G F R$-2. We investigated whether $\mathrm{VEGF}_{165}$ induced a more invasive phenotype on leukemic cells. This was demonstrated using a migration system in which transwell inserts were coated with a thin layer of Matrigel, a model of invasion through the basement membrane. VEGF $_{165}$ induced trans-basement membrane migration of HL-60 cells and primary leukemias (Figure 6). This process requires MMP production and activation, as the VEGF-induced cell migration was blocked by recombinant human TIMP-1 (Figure 6). TIMP-1 had no effect on leukemic cell migration through bare (uncoated) transwells (data not shown).

As shown here, the mitogenic effects of $\mathrm{VEGF}_{165}$ on leukemic cells were mediated through VEGFR-2. However, in the migration system used in our studies, incubation of HL-60 cells with IMC-1C11 (at $1 \mu \mathrm{g} / \mathrm{mL}$ ) could only partially (40\%) block VEGF 165 -induced migration through Matrigel (Figure 6). On the other hand, an mAb to VEGFR-1, used at the same concentration, significantly blocked (60-70\%) HL-60 cell migration (Figure 6; $P<0.05$ ), suggesting that $\mathrm{VEGF}_{165}$ may induce MMP activation and cell migration by interacting with both receptors. In fact, incubation of HL-60 cells with VEGFR-1 mAb and IMC-1C11 blocked $\mathrm{VEGF}_{165}$-induced migration more effectively (70-80\%) than either antibody alone (Figure 6). On primary leukemias, both VEGFR-1 mAb and IMC-1C11

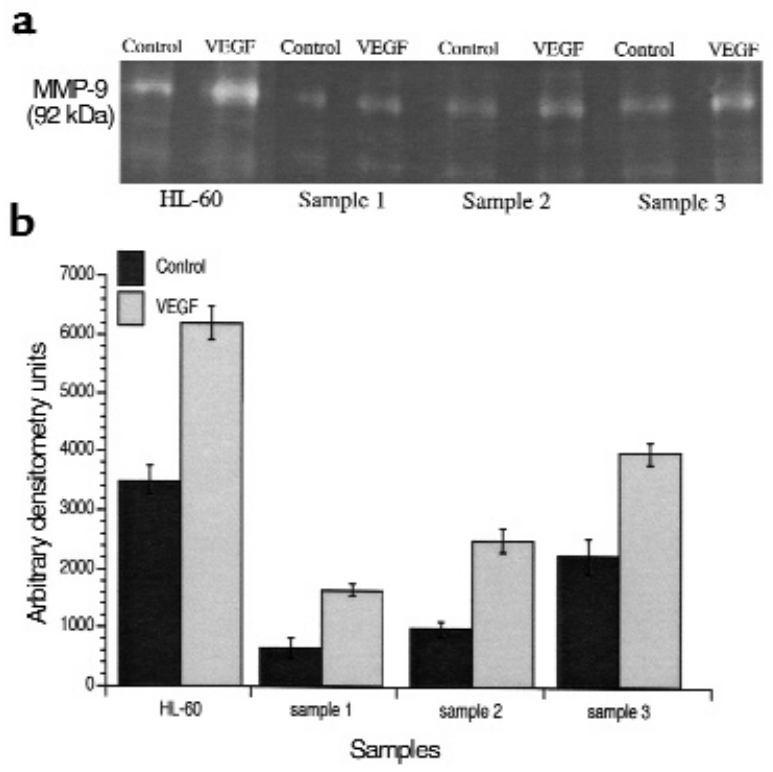

Figure 5

$\mathrm{VEGF}_{165}$ induces MMP secretion by leukemic cells. (a) Zymographic analysis of leukemic cell supernatants, with or without VEGF 165 stimulation for 18 hours. (b) Quantification of the gelatinolytic activity detected on the culture supernatants. Incubation of leukemic cells with VEGF 165 had a significant $(P<0.05)$ effect on the level of MMP production in vitro, as detected by zymography. The results shown are representative of three independent experiments. 


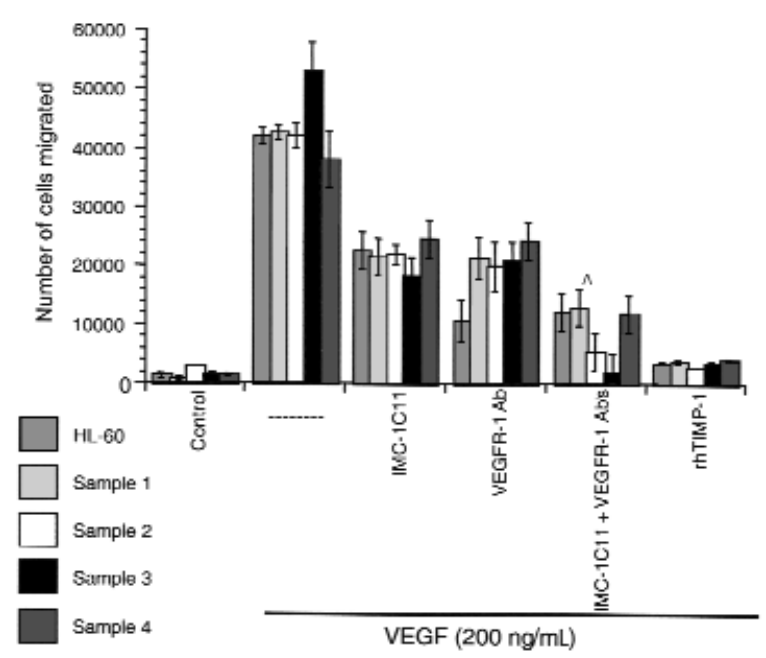

Figure 6

$V_{E G F_{165}}$ induces leukemic cell migration through Matrigel-coated transwells, a process mediated through VEGFR-2 and VEGFR-1. Migration of HL-60 cells and four primary leukemias through Matrigel-coated transwells, in response to $200 \mathrm{ng} / \mathrm{mLVEGF}_{165}$, is shown. This process requires MMP activity, as shown by the effects of rhTIMP-1. IMC-1C11 partially blocked leukemic cell migration, but incubation of the cells with VEGFR-1 mAb and IMC-1C11 was more effective than either antibody alone $\left({ }^{A} P<0.05\right)$. These results are representative of three independent experiments.

showed, overall, comparable migration-blocking effects (Figure 6). As shown for the HL-60 cells, incubation of primary leukemias with both antibodies was more effective than either antibody alone, blocking $\mathrm{VEGF}_{165^{-}}$ induced migration by $80 \%$ (Figure 6).

Leukemic cells release VEGF in vivo. Using NOD-SCID mice as an in vivo model, we investigated whether VEGFR-2-positive leukemias, HL-60, HEL, and a primary leukemia, released VEGF in vivo. As shown in Figure 7a, human VEGF plasma levels increased 7 and 14 days after HL-60 cell injection. Importantly, murine VEGF plasma levels remained very low (at or below the assay detection level; Figure 7a) throughout the experiment. Similar results were obtained using the HEL cell line (data not shown) and a primary leukemia sample (Figure 7b). If left untreated, mice injected with this primary leukemia had increased circulating human VEGF levels 14 days after inoculation (Figure 7b). However, similarly to the HL-60 model, murine VEGF plasma levels did not increase in these mice (data not shown).

IMC-1C11 blocks lenkemic growth in vivo. In the absence of any treatment, or treated with unrelated human IgG, mice injected with $3 \times 10^{6} \mathrm{HL}-60$ cells (intravenously) survived only $14-25$ days ( $n=8$ for each treatment), highlighting the aggressive nature of these leukemic cells. However, mice treated with IMC-1C11 survived five times longer than untreated mice (Figure 8a; $P$ < 0.005 ) and, as shown in Figure 7a, had reduced human VEGF plasma levels throughout the experiment. Similarly, untreated HEL-inoculated mice died within 45 days, whereas those treated with IMC-1C11 survived significantly longer $(P<0.05$; Figure $8 b)$.
These observations were extended to include a primary leukemia. In the absence of any treatment, mice inoculated with $5 \times 10^{6}$ primary leukemic cells died within 3 weeks. However, as shown for the two leukemic cell lines, IMC-1C11 prolonged the survival of primary leukemia-bearing mice (Figure 8c). This increase in survival was accompanied by a reduction in human VEGF plasma levels (Figure 7b). Given that IMC-1C11 is specific for human VEGFR-2 (KDR) and does not cross react with murine flk-1, the data suggest that treatment of inoculated mice with IMC-1C11 blocks leukemic growth by impeding leukemia-derived VEGF from interacting with VEGFR-2 on leukemic cells.

Decreased metastasis in mice treated with IMC-1C11. As shown in vitro, VEGF can promote an invasive phenotype on VEGFR-2-positive leukemic cells, which may be one mechanism by which extramedullary leukemic masses are established. Using the in vivo models described here, it was next investigated whether VEGFR-2 blockade affected metastatic behavior.

In untreated HL-60 inoculated mice, histological analysis of the liver and spleen revealed the presence of metastatic lesions in both organs (Figure 9, b and d). Liver sections had several areas occupied by leukemic cells, with signs of active cellular proliferation (Figure $9 \mathrm{~b})$. Furthermore, the white pulp area of the spleen of these mice was largely replaced by leukemic cells (Figure $9 \mathrm{~d}$ ). However, 14 days after start of the experiment,

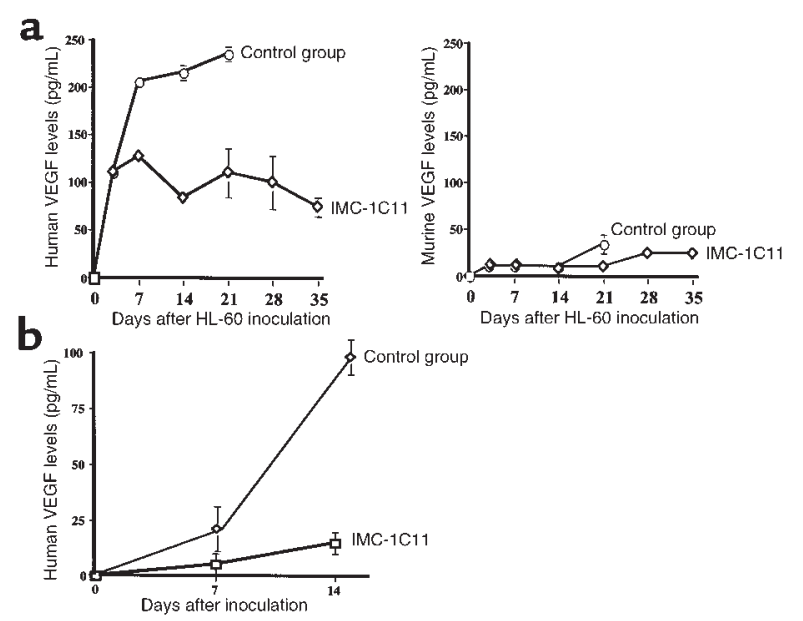

Figure 7

(a) HL-60 injection into NOD-SCID mice induces high levels of human but not murine VEGF in mouse plasma. Mice were injected with $3 \times 10^{6} \mathrm{HL}-60$ cells (intravenously), and the human and murine VEGF plasma levels were determined at different time points after injection. Mice treated with IMC-1C11 had reduced circulating human VEGF levels $(P<0.01)$. The results shown are representative of three mice per time point and of two separate experiments. (b) Inoculation of a primary leukemia into NODSCID mice increased human VEGF levels in mouse plasma. Mice were injected with $5 \times 10^{6}$ leukemic cells (intravenously) and the human VEGF plasma levels were quantified by ELISA, as described in Methods. Mice treated with IMC-1C11 had reduced levels of circulating human VEGF $(P<0.05)$. The results shown are representative of three mice per time point. 
IMC-1C11-treated mice had normal liver and spleen histology (Figure 9, a and c). In these mice, there was no evidence of metastatic lesions in the liver (Figure 9a), and the spleen appeared normal, with evidence of apoptosis (Figure 9c). Mice inoculated with the HEL cell line showed similar results. In control mice, 15 days

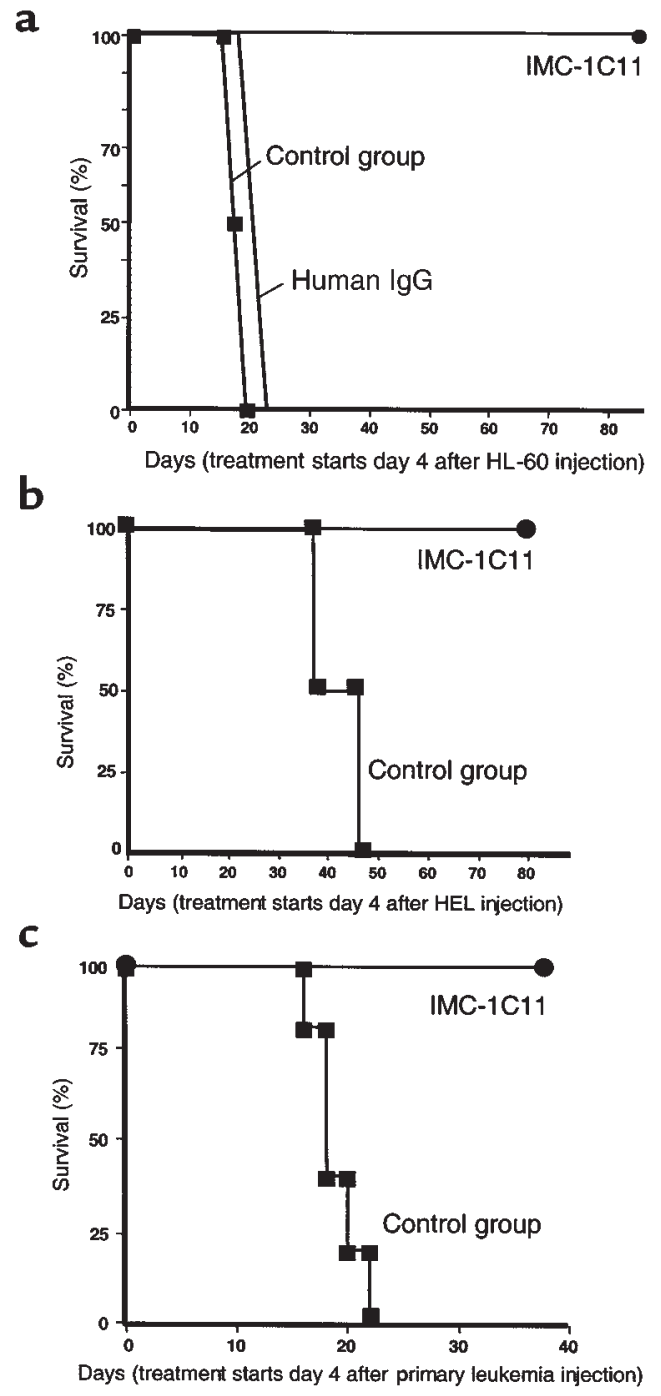

\section{Figure 8}

(a) Mouse survival (\%) after intravenous HL-60 injection. Mice were injected with $3 \times 10^{6}$ cells intravenously, and 4 days after leukemic cell injection they were left untreated (control, $n=8)$, or treated $(n=$ $8)$ intraperitoneally with IMC-1C11 $(400 \mu \mathrm{g} /$ dose $)$ or with an unrelated human $\operatorname{lgG}(n=8,400 \mu \mathrm{g} / \mathrm{dose})$ three times a week. The results shown are representative of three separate experiments. (b) Mouse survival (\%) after intravenous injection of HEL cells. Mice were injected with $5 \times 10^{6}$ cells intravenously, and 4 days after leukemic cell injection they were treated with PBS/BSA $(n=8)$ or with IMC-1C11 $(n=8$, intraperitoneal injection, $400 \mu \mathrm{g} / \mathrm{dose})$, three times a week. The results shown are representative of two separate experiments. (c) Mouse survival (\%) after intravenous injection of primary human leukemia cells. Mice were injected with $5 \times 10^{6}$ cells intravenously, and 4 days after leukemic cell injection were treated with PBS/BSA $(n=8)$ or with IMC-1C11 $(n=8$, intraperitoneal injection, 400 $\mu \mathrm{g} /$ dose), three times a week. The results shown are representative of two separate experiments. after the start of the experiment, there were numerous metastatic foci in the spleen and lungs (more than three metastatic foci seen at $10 \times$ magnification). However, mice treated with IMC-1C11 had no evidence of metastasis (data not shown).

Decreased circulating leukemic cells and reduced bone marrow lenkemic cell engraftment in mice treated with IMC1C11. In HL-60-injected mice, untreated or treated with unrelated human IgG, 15 days after the start of the experiment, there were $6 \%$ human $\mathrm{CD} 15$-positive cells in the bone marrow, of which approximately $4 \%$ were VEGFR-2 positive (Figure 10a). However, mice treated with IMC-1C11 had tenfold less human cells in the bone marrow (Figure 10a). Similar results were obtained with the HEL leukemia cell line (Figure 10a). These cells, contrarily to HL-60, do not express CD15, but express VEGFR-2/KDR. As shown in Figure 10a, 15 days after the start of the experiment, there were tenfold more VEGFR-2-positive HEL cells in the bone marrow of untreated mice compared with those treated with IMC-1C11 $(P<0.005)$.

We also quantified the number of circulating leukemic cells in mice inoculated with a primary leukemia. Fourteen days after the start of the experiment, untreated mice had $12 \%$ circulating CD 45+VEGFR-2+ leukemic cells (Figure 10b). However, those treated with IMC-1C11, at the same time point, had less than $1 \%$ circulating leukemic cells (Figure 10b). Similar results were obtained with the two cell lines HL60 and HEL (data not shown).

\section{Discussion}

It is now well established that most human leukemic cell lines as well as primary isolated leukemias have the capacity to elaborate VEGF $(2,3)$. Several studies have also demonstrated expression of VEGFR-2 in certain leukemias $(3,4)$, and one recent study correlated the levels of VEGF production by lymphoid cells with their ability to form subcutaneous tumors in vivo (17).

In this report, we demonstrate that primary leukemias and leukemic cell lines produce VEGF, and subsets of human acute leukemias express functional VEGF receptors, namely, VEGFR-2. On VEGFR2-positive leukemias, blockade of VEGF binding to its receptor by a neutralizing $\mathrm{mAb}$ to VEGFR-2, IMC$1 \mathrm{C} 11$, resulted in decreased leukemic cell growth in serum-free conditions, suggesting the existence of an autocrine loop between leukemia-derived VEGF and its VEGFR-2. Moreover, as shown for endothelial cells, addition of exogenous VEGF induced VEGFR-2 phosphorylation and proliferation of subsets of acute leukemias primarily by signaling through VEGFR-2. VEGF also induced MMP production and promoted trans-basement membrane migration of leukemic cells by interacting with VEGFR-2 and VEGFR-1. This study provides the first demonstration that blockade of VEGF/VEGFR-2 interactions decreases leukemic cell growth in serum-free (in the absence of exogenous growth factors) conditions. It also shows for the first 
time that VEGF receptors expressed by leukemic cells are functional, signaling receptors, conveying signals similar to those induced on endothelial cells.

To define the role of VEGF/VEGFR-2 autocrine loop in the regulation of leukemic cell growth in vivo, we have used a well-established xenotransplantation model in which human leukemic cells are engrafted into sublethally irradiated immunodeficient (NODSCID) mice (18-20). We used three VEGFR-2-positive leukemias, the HL-60 and HEL cell lines and a primary leukemia sample, for these experiments. Injection of leukemic cells into NOD-SCID mice resulted in the engraftment and formation of leukemic foci in the bone marrow, spleen, liver, and peripheral circulation of NOD-SCID mice. We demonstrate that leukemic cells produce human VEGF that could be detected at high levels in the plasma of leukemia xenotransplanted mice, as determined by a human specific VEGF ELISA. Rising levels of human VEGF were detected in plasma of the xenotransplanted mice and increased over time as the leukemic cells proliferated in the NOD-SCID mice. In contrast, plasma levels of murine VEGF produced were insignificant and did not increase during in vivo proliferation of any of the cell lines or primary sample tested.

In the absence of any intervention, xenografted NOD-SCID mice succumb to metastatic proliferation of leukemic cells within 2-3 weeks of inoculation. However, treatment of inoculated mice with IMC-1C11, inhibited proliferation of HL-60, HEL, and primary leukemic cells in NOD-SCID mice and promoted their long-term survival. Histological examination of liver and spleen demonstrated that treatment of xenotransplanted mice with IMC-1C11 effectively inhibits proliferation and establishment of leukemic infiltrates. In treated mice, the number of VEGFR-2+ leukemic cells in the bone marrow and peripheral blood was also dramatically reduced. Furthermore, a reduction in leukemic cells and subsequent increase in mouse sur- vival was accompanied by a decrease in circulating human VEGF levels. These data strongly suggest that endogenously produced human VEGF by leukemic cells promotes their growth, survival, and capacity to metastasize through interaction with human VEGFR2. Collectively, these data illustrate the plasticity of VEGFRs signaling within the vascular and hematopoietic lineages and their potential to support the proliferation certain subsets of VEGFR-2+ $2^{+}$acute leukemias.

In humans, acute leukemias are classified based on progenitor cell type and differentiation state of the stem cell they originated from (M1 to M7). The majority of leukemias are due to malignant transformation of the myeloid progenitors (M1, M2, M3, M4, M5), which are mostly localized to the bone marrow and the peripheral circulation. However, leukemic cells occasionally acquire the capacity to invade various tissues, setting up niches for the formation of large tumor masses referred to as chloromas. Based on our in vivo data, most of the human chloroma samples tested to date express VEGFR-2 and VEGFR-1. Given that formation of chloromas, similar to vessel formation, requires sequential invasion, proliferation, and establishment of a neovasculature, it is not surprising that leukemic cells with the potential to form chloromas produce VEGF and may also express VEGFRs. In this regard, it has been recently reported that the levels of VEGF produced by lymphoid cells are strictly correlated with their ability to form subcutaneous tumors in vivo (17). For instance, HL-60 cells release VEGF, express VEGF receptors, and represent one leukemic model with the capacity to metastasize and to form a solid, vascularized tumor if implanted subcutaneously. Therefore, leukemias destined to form chloromas may utilize VEGFRs to upregulate the expression of MMPs and facilitate invasion into the tissues. Expression of VEGFR-2 may subsequently promote leukemic cell proliferation. In the absence of counterregulatory factors, the leukemias may continue to proliferate.

\section{Figure 9}

Histology of liver (a and $\mathbf{b}$ ) and spleen (c and $\mathbf{d}$ ) from HL-60-injected mice. Untreated (control) mice had evidence of metastatic disease in the liver (white and red arrows, b) and spleen (d). In contrast, mice treated with IMC-1C11 showed no evidence of leukemic cells in the liver (a), and the spleen had evidence of apoptosis (blue arrows, c) but normal histology.
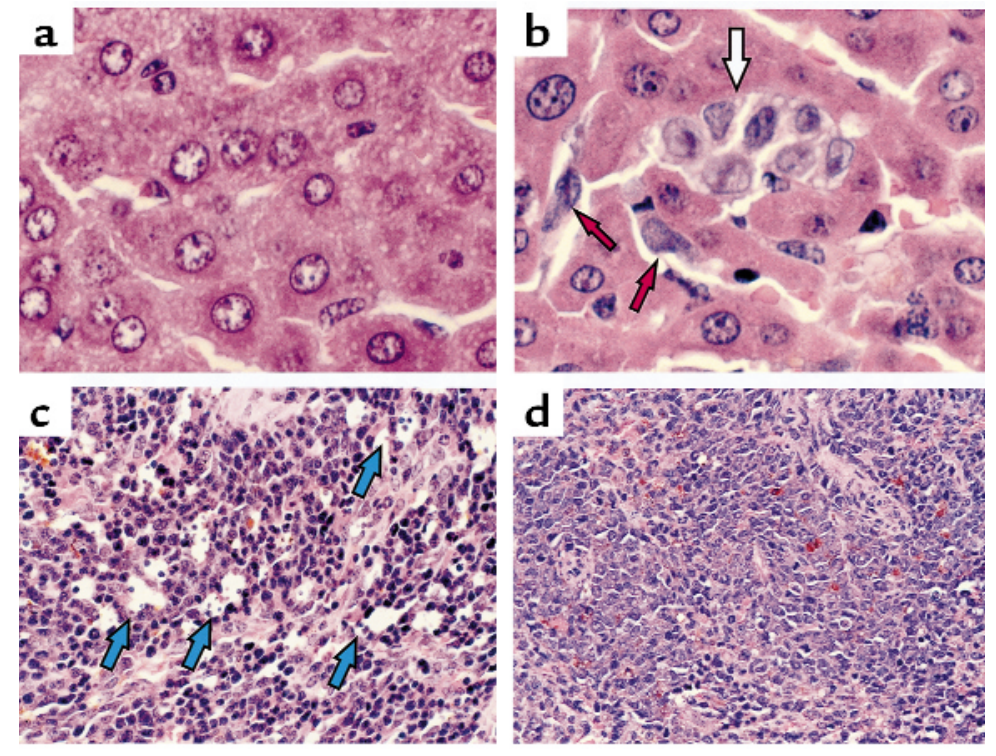

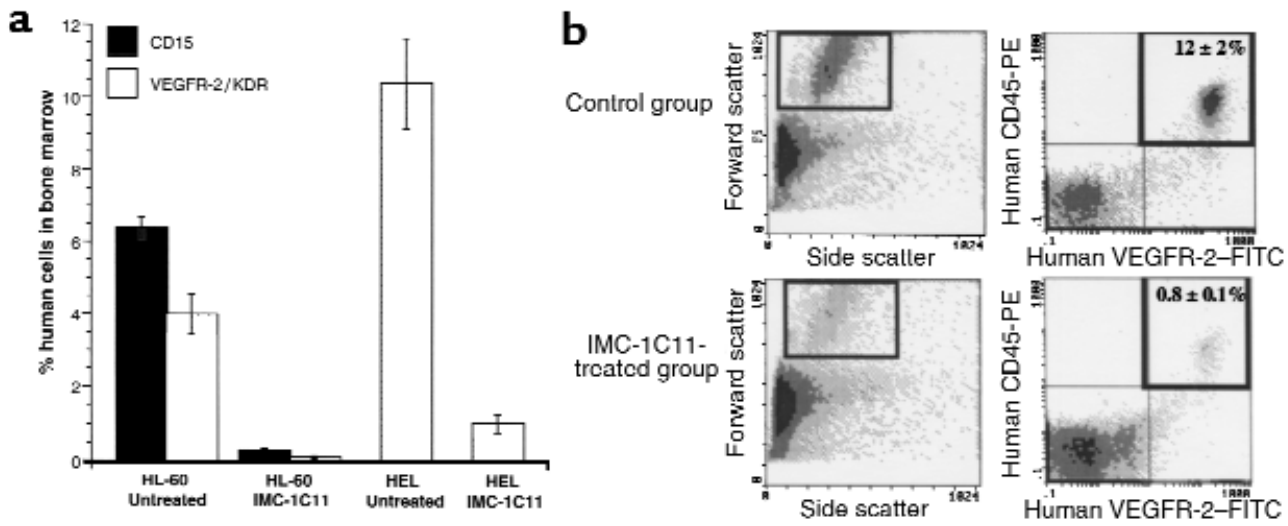

\section{Figure 10}

(a) Leukemic cell engraftment (\%) in the bone marrow of HL-60- and HEL-inoculated mice. Day 15 after the start of the experiment, bone marrow cells from inoculated mice were stained for human CD15 and human VEGFR-2/KDR and quantified by flow cytometry. IMC1 1C11-treated mice had a significantly lower percentage of human cells in the bone marrow than untreated mice $(P<0.001)$. These results are representative of three mice per cell line. (b) Quantification of primary leukemic cells in the peripheral blood of inoculated mice. PBMCs from primary leukemia-inoculated mice were stained for human CD45 and human VEGFR-2 and quantified by flow cytometry, as described in Methods. Fourteen days after the start of treatment, IMC-1C11-treated mice had significantly less circulating CD45 $V E G F R-2^{+}$human leukemic cells than untreated mice $(P<0.05)$. These results are representative of three mice.

Under physiological conditions, most tissues have the capacity to produce VEGF. Elevated levels of VEGF have been detected in the peripheral circulation of patients with various malignancies $(21,22)$. Therefore, it is conceivable that increases in VEGF secretion into the peripheral circulation of leukemic patients may result in mobilization of leukemic cells to the bone marrow extravascular space.

Malignant transformation of leukemic cells may be associated with the acquisition of specific tyrosine kinases that may be involved in the self-renewal process. Therefore, reactivation of VEGFR-2 may be one mechanism by which certain subsets of leukemic cells establish their autonomous growth. Recent demonstration that VEGFR-2 signaling is critical for hematopoietic stem cell proliferation underscores

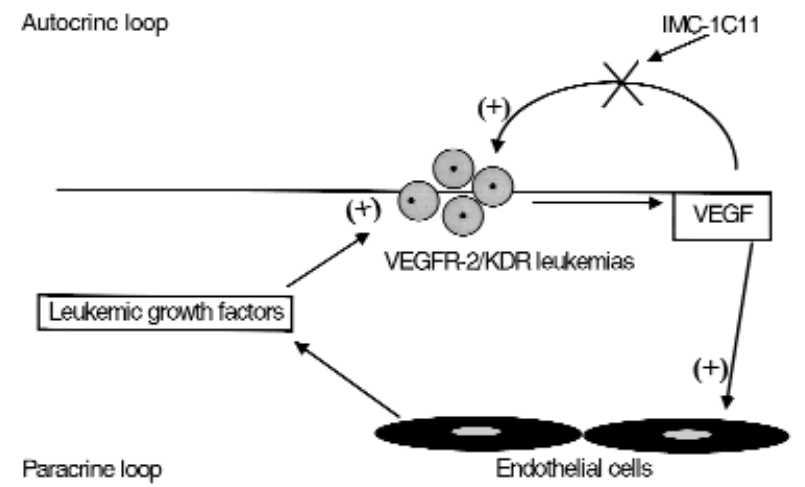

Figure 11

On VEGFR-2-positive leukemias, VEGF may support leukemic cell growth through paracrine (by increasing bone marrow endothelium cell mass) and autocrine (supporting leukemic cell survival) mechanisms. Antibodies against human VEGFRs may block the autocrine loop induced by leukemia-derived human VEGF. the significance of VEGFR-2 in leukemic cell proliferation. Other studies have also shown that solid tumors such as breast, ovarian, and prostate carcinomas express VEGFR-2 mRNA (23-25). However, activation of VEGFR-2 has not been shown to convey any physiological signal in these malignancies possibly due to lack of downstream signaling pathways in these cells. Because CD34+VEGFR-2+ stem cells, as result of oncogenic transformation, may give rise to acute leukemias, it is not surprising that VEGFR-2 is functional on these cells.

It is now well established that growth of solid tumors is angiogenesis dependent (26). A previous report also suggested that progression of leukemias may be associated with increases in bone marrow endothelial cell mass (27). This process may be mediated through the release of VEGF, which will induce endothelial proliferation and stimulate the release of hematopoietic growth factors to support leukemic cell growth (2). In the present report, it is hypothesized that in liquid tumors such as leukemias, VEGF may serve two functions, one to enhance endothelial cell proliferation and the production of hematopoietic growth factors and another to promote leukemic cell growth through an autocrine loop (Figure 11). Based on our in vivo data, the growth of leukemic cells xenotransplanted into NOD-SCID mice was inhibited by human specific neutralizing $\mathrm{mAb}$ to VEGFR-2, suggesting that an autocrine loop generated by VEGF/VEGFR-2, in addition to paracrine loops, may mediate leukemic cell proliferation (Figure 11).

Recent studies have shown that leukemic cells are capable of producing other forms of VEGF including VEGF-C (28) and possibly VEGF-D. VEGF-C and VEGF-D signal through both VEGFR-2 and VEGFR-3 (Flt-4). However, VEGF (VEGF-A) as well as VEGF-C 
and VEGF-D promote the proliferation of the target cells primarily through VEGFR-2. Therefore, inhibition of VEGRF-2 signaling may provide an effective means of blocking the endogenous signaling induced by various forms of VEGFs.

Using mAb's that could differentially block murine or human VEGFR-2 and various forms of VEGFs may ultimately define the relative significance of the autocrine versus the paracrine role of VEGF in supporting the growth of VEGF-dependent leukemias, in particular those that have the capacity to form chloromas. These studies may lay the foundation for a novel therapeutic approach using a combination of mAb's to VEGF and VEGFRs for the treatment of VEGFR-2+ leukemias.

\section{Acknowledgments}

S. Rafii is supported by National Heart, Lung, and Blood Institute (NHLBI) grants R01 HL-58707 and R01 HL-61849, an American Heart Association GrantIn-Aid, the Dorothy Rodbell Foundation for Sarcoma Research, and the Rich Foundation. M.A.S. Moore is supported by NHLBI grant R01 HL-61401 and the Gar Reichman Fund of the Cancer Research Institute.

1. Ziegler, B.L., et al. 1999. KDR receptor: a key marker defining hematopoietic stem cells. Science. 285:1553-1558.

2. Fiedler, W., et al. 1997. Vascular endothelial growth factor, a possible paracrine growth factor in human acute myeloid leukemia. Blood. 89:1870-1875.

3. Bellamy, W.T., Richter, L., Frutiger, Y., and Grogan, T.M. 1999. Expression of vascular endothelial growth factor and its receptors in hematopoietic malignancies. Cancer Res. 59:728-733.

4. Katoh, O., Tauchi, H., Kawaishi, K., Kimura, A., and Satow, Y. 1995. Expression of the vascular endothelial growth factor (VEGF) receptor gene, KDR, in hematopoietic cells and inhibitory effect of VEGF on apoptotic cell death caused by ionizing radiation. Cancer Res. 55:5687-5692.

5. Klagsbrun, M., and D'Amore, P.A. 1996. Vascular endothelial growth factor and its receptors. Cytokine Growth Factor Rev. 7:259-270.

6. Ortega, N., et al. 1997. Systemic activation of the vascular endothelial growth factor receptor KDR/flk-1 selectively triggers endothelial cells with an angiogenic phenotype. Am. J. Pathol. 151:1215-1224.

7. Shalaby, F., et al. 1995. Failure of blood-island formation and vasculogenesis in Flk-1-deficient mice. Nature. 376:62-66.

8. Fong, G.H., Rossant, J., Gertsenstein, M., and Breitman, M.L. 1995. Role of the Flt-1 receptor tyrosine kinase in regulating the assembly of vascular endothelium. Nature. 376:66-70.

9. Skobe, M., Rockwell, P., Goldstein, N., Vosseler, S., and Fusenig, N.E. 1997. Halting angiogenesis suppresses carcinoma cell invasion. Nat. Med.
3:1222-1227.

10. Prewett, M., et al. 1999. Antivascular endothelial growth factor receptor (fetal liver kinase 1) monoclonal antibody inhibits tumor angiogenesis and growth of several mouse and human tumors. Cancer Res. 59:5209-5218.

11. Millauer, B., Shawver, L.K., Plate, K.H., Risau, W., and Ullrich, A. 1994. Glioblastoma growth inhibited in vivo by a dominant-negative Flk-1 mutant. Nature. 367:576-579.

12. Strawn, L.M., et al. 1996. Flk-1 as a target for tumor growth inhibition. Cancer Res. 56:3540-3545.

13. Zhu, Z., et al. 1998. Inhibition of vascular endothelial growth factorinduced receptor activation with anti-kinase insert domain-containing receptor single-chain antibodies from a phage display library. Cancer Res. 58:3209-3214.

14. Lu, D., et al. 2000. Identification of the residues in the extracellular region of KDR important for interaction with vascular endothelial growth factor and neutralizing anti-KDR antibodies. J. Biol. Chem. 275:14321-14330.

15. Leber, T.M., and Balkwill, F.R. 1997. Zymography: a single-step staining method for quantitation of proteolytic activity on substrate gels. Anal. Biochem. 249:24-28.

16. Hamada, T., et al. 1998. Transendothelial migration of megakaryocytes in response to stromal cell-derived factor 1 (SDF-1) enhances platelet formation. J. Exp. Med. 188:539-548.

17. Fusetti, L., et al. 2000. Human myeloid and lymphoid malignancies in the non-obese diabetic/severe immunocompromised mouse model: frequency of apoptotic cells in solid tumors and efficiency and speed of engraftment correlate with vascular endothelial growth factor production. Cancer Res. 60:2527-2534.

18. Clutterbuck, R.D., et al. 1998. Inhibitory effect of simvastatin on the proliferation of human myeloid leukaemia cells in severe combined immunodeficient (SCID) mice. Br. J. Haematol. 102:522-527.

19. Terpstra, W., et al. 1995. Conditions for engraftment of human acute myeloid leukemia (AML) in SCID mice. Lenkemia. 9:1573-1577.

20. Machado, E.A., et al. 1984. Proliferation and differentiation of human myeloid leukemic cells in immunodeficient mice: electron microscopy and cytochemistry. Blood. 63:1015-1022.

21. Salven, P., Manpaa, H., Orpana, A., Alitalo, K., and Joensuu, H. 1997. Serum vascular endothelial growth factor is often elevated in disseminated cancer. Clin. Cancer Res. 3:647-651.

22. Obermair, A., et al. 1998. Concentration of vascular endothelial growth factor (VEGF) in the serum of patients with suspected ovarian cancer. Br. J. Cancer. 77:1870-1874

23. Kranz, A., Mattfeldt, T., and Waltenberger, J. 1999. Molecular mediators of tumor angiogenesis: enhanced expression and activation of vascular endothelial growth factor receptor KDR in primary breast cancer. Int. J. Cancer. 84:293-298.

24. Abu-Jawdeh, G.M., et al. 1996. Strong expression of vascular permeability factor (vascular endothelial growth factor) and its receptors in ovarian borderline and malignant neoplasms. Lab. Invest. 74:1105-1115.

25. Ferrer, F.A., et al. 1999. Expression of vascular endothelial growth factor receptors in human prostate cancer. Urology. 54:567-572.

26. Folkman, J. 1997. Angiogenesis and angiogenesis inhibition: an overview. EXS. 79:1-8.

27. Perez-Atayde, A.R., et al. 1997. Spectrum of tumor angiogenesis in the bone marrow of children with acute lymphoblastic leukemia. Am. J. Pathol. 150:815-821.

28. Fielder, W., et al. 1997. Expression of FLT4 and its ligand VEGF-C in acute myeloid leukemia. Leukemia. 11:1234-1237. 